\title{
ANÁLISE DO NÍVEL DE EFICÁCIA DA PULVERIZAÇÃO INTRA- DOMICILIÁRIA NO COMBATE À MALÁRIA NO BAIRRO 7 DE SETEMBRO - CHIMOIO (2018-2019)
}

Orlando Momade Ossufo ${ }^{1}$, Elaine da Silva Reis ${ }^{2}$, Chennyfer Dobbins Abi Rached ${ }^{3}$

1 Especialista em Saúde Pública, Faculdade Unyleya. Brasília - Brasil - Email: São Paulo - Brasil - email: orlandopdf@gmail.com

2 Graduanda de Enfermagem. Universidade Nove de Julho - UNINOVE. São Paulo Brasil - Email: São Paulo - Brasil - email: elainesr30@gmail.com

3 Doutora em Saúde Coletiva; Mestre em Economia da Saúde pela Universidade Federal de São Paulo (UNIFESP); Docente do Programa de Mestrado Profissional em Gestão em Sistemas de Saúde - Universidade Nove de Julho - UNINOVE. São Paulo Brasil email: chennyferr@yahoo.com.br ORCID: https://orcid.org/0000-0002-44993716

\section{RESUMO}

Para uma melhor percepção e análise, este trabalho é estruturado por capítulos e, portanto, este capítulo contém três capítulos. Sendo que, no primeiro capítulo é apresentada a introdução, os objetivos do estudo: geral e específico que se espera alcançar e a justificação. Em seguida, são apresentadas a definição do problema de pesquisa, a hipótese de estudo e a delimitação do estudo. No segundo capítulo é apresentada a revisão da literatura, onde diferentes autores apresentam os principais delineamentos sobre a pulverização intra-domiciliar. No terceiro capítulo é apresentada a metodologia de pesquisa, onde se apresenta a classificação da pesquisa, fontes de dados, universo e amostra, dados de pesquisa, plano de coleta de dados e finalmente instrumentos que serão utilizados para a coleta de dados. O objetivo geral deste trabalho é analisar o nível de eficácia da pulverização intra-domiciliária na luta contra a malária no 7 de Setembro bairro de Chimoio. Em vista do nosso objetivo geral, focamos os específicos: identificar o nível de conhecimento das vantagens e desvantagens da pulverização intra-domiciliar, pelos moradores do $7^{\circ}$ de September Chimoio Neighborhood; Descreve o nível de práticas sobre a malária no $7^{\circ}$ de September Chimoio bairro; Identifica fatores que influenciam a rejeição da pulverização intra-domiciliária no bairro 7 de setembro-Chimoio.

Palavras-chave: Nível de eficácia; Pulverização intra-domiciliária; Luta contra a malária.

\begin{abstract}
For a better perception and analysis, this work is structured by chapters and so this chapter contains three chapters. Being that, in the first chapter is presented the introduction, the objectives of the study: general and specific that are expected to reach and the justification. Then, the definition of the research problem, the hypothesis of study and the
\end{abstract}


delimitation of the study are presented. In the second chapter the literature review is presented, where different authors present the main delineations about intra-household spraying. In the third chapter the research methodology is presented, where the classification of the research, data sources, universe and sample, research data, the data collection plan and finally instruments that will be used for the data collection are presented. The general objective of this work is to analyze the Efficacy Level of Intradomiciliary Spraying in the Fight against Malaria in the 7th of September-Chimoio Neighborhood. In view of our general objective, we focus the specific ones: Identify the level of knowledge of the advantages and disadvantages on Intra-Domiciliary Spraying, by the residents of the 7th of September Chimoio Neighborhood; Describe the level of practices on malaria in the 7th of September Chimoio Neighborhood; Identify factors that influence the rejection of Intra-Domiciliary Spraying in the 7 de Setembro-Chimoio neighborhood.

Keywords: Level of Efficacy; Intra-domiciliary spraying; Fight against malaria.

\section{INTRODUÇÃO}

A saúde está sempre ligada ao ambiente, pois é difícil apresentar um estado de saúde favorável num contexto ambiental precário e desfavorável. Libinga (2011), aponta que as condições precárias de habitação, a falta de condições e os meios para combate, a inobservância dos princípios básicos de saneamento ambiental são a razão do aumento de casos de malária.

Além disso, Ribeiro e Rooke (2010), referem que os estudos do Banco Mundial (1993) estimam que o meio ambiente inadequado é responsável por maior parte da ocorrência de doenças em países em desenvolvimento.

Corroborando com a mesma afirmação, a Organização Mundial da Saúde - OMS (2009), considera que a malária é um dos grandes problemas de saúde pública nos países em vias de desenvolvimento, e estima-se que 300 a 500 milhões de pessoas sejam infetadas a cada ano e que nos países da África estão concentradas $90 \%$ dessas pessoas. Apesar dos esforços cada vez crescentes desenvolvidos pelo Governo de Moçambique na redução da morbilidade e mortalidade devido à malária no país, a doença continua a ser uma das principais causas de morte, sendo responsável por cerca de $40 \%$ de todas as consultas externas, $60 \%$ de admissões às enfermarias de pediatria e $30 \%$ de mortes nos hospitais. Em Moçambique, os principais vetores da malária são endofágicos 
(alimentam-se no interior das casas) e, endofílicos (repousam dentro das casas), facto que torna estes vetores vulneráveis à pulverização intra-domiciliária com o uso de inseticidas (MISAU, 2006).

Em Moçambique, a malária é a principal causa de problemas de saúde, sendo responsável por $40 \%$ de todas as consultas externas. Até $60 \%$ de doentes internados nas enfermarias de pediatria são admitidos como resultado da malária severa. A malária é também a principal causa de mortalidade nos hospitais em Moçambique, ou seja, de quase $30 \%$ de todos os óbitos registados. A estimativa de prevalência no grupo etário de 2 a 9 anos de idade varia de 40 a $80 \%$, com $90 \%$ de crianças menores de 5 anos de idade infetadas por parasitas da malária em algumas áreas (PNCM, 2007).

A malária continua a ser a doença causadora de mais mortes e responsável pelo maior número de internamentos hospitalares na província central de Manica, tendo provocado em 2014, 213 óbitos, de um total de 370 mil doentes que procuraram as unidades sanitárias em busca de assistência médica (DPS-Manica).

Tem sido frequente no bairro 7 de Setembro, a recusa por parte dos moradores, quando chega a época de pulverização das residências deste bairro, nota-se que os moradores deste bairro tem se recusado a ceder espaço para os agentes de pulverização intradomiciliária poderem proceder com as suas atividades.

Um relatório anual da Organização Mundial de Saúde (OMS) sobre a malária, divulgado em finais de 2016, estima que em Moçambique houve uma redução de casos de malária entre 2010 e 2015, assim como da sua letalidade graças ao uso da rede mosquiteira e a pulverização intra-domiciliária.

\section{MÉTODOS}

A revisão bibliográfica constituiu no levantamento dos conteúdos teóricos em diversas referências bibliográficas que abordam sobre a temática e os diversos aspetos inerentes a ela. Onde posteriormente faz-se leitura, análise e compilação dos mesmos compondo a parte teórica do trabalho.

A consulta bibliográfica consistiu na leitura de algumas obras relacionadas ao tema, reforçada pela informação documentada sobre casos de malária no bairro 7 de Setembro e a aceitabilidade da Pulverização Intra-domiciliária. 
O presente estudo, circunscreve-se na abordagem de análise da influência da pulverização intra-domiciliária no bairro 7 de setembro - Chimoio, onde iremos buscar compreender até que ponto este processo exerce um certo impacto aos moradores deste bairro.

E, insere-se na especialidade da Saúde Pública, onde os aspetos abordados são o impacto da pulverização intra-domiciliária, e sua possível influência no conforto correlação ao combate à malária.

\section{RESULTADOS}

A malária é uma doença que preocupa as autoridades de saúde e a população em geral. Existem no país várias instituições e organizações engajadas na luta contra a doença, mas ela continua sendo uma das doenças responsáveis por internamentos e mortes hospitalares (Libinga, 2011).

A malária é endémica em todo o país, nas áreas onde o clima favorece a sua transmissão ao longo de todo o ano, atingindo o seu ponto mais alto após a época chuvosa (Dezembro à Abril).

A intensidade da transmissão varia de ano para ano e de região para região, dependendo da precipitação, altitude e temperaturas. Algumas das áreas secas do país são tidas como propensas à epidemia. O Plasmodium falciparum é o parasita mais frequente, sendo responsável por cerca de $90 \%$ de todas infeções maláricas, enquanto que o P. malariae e o P. ovale são responsáveis por 9.1 e $0,9 \%$ de todas infeções, respetivamente. (Libinga, 2011).

Segundo a análise situacional realizada no ano 2000, em Moçambique, a malária é a principal causa de problemas de saúde, sendo responsável por $40 \%$ de todas as consultas externas. Até $60 \%$ de doentes internados nas enfermarias de pediatria são admitidos como resultado da malária severa. A malária é também a principal causa de mortalidade nos hospitais em Moçambique, ou seja, de quase 30\% de todos os óbitos registados. (MISAU, 2009)

A estimativa de prevalência no grupo etário de 2 a 9 anos de idade varia de 40 a $80 \%$, com $90 \%$ de crianças menores de 5 anos de idade infetadas por parasitas da malária em algumas áreas. (MISAU, 2009) 
O acesso aos cuidados de saúde em Moçambique é muito baixo e estima-se que $50 \%$ da população vive a mais de 20 quilómetros da mais próxima unidade sanitária, uma situação que efetivamente implica não haver acesso aos serviços de saúde para uma grande parte da população. A malária é também o maior problema que afeta mulheres grávidas nas zonas rurais.

Aproximadamente $20 \%$ das mulheres grávidas está infetada pelo parasita, sendo as primigestas as mais afetadas com uma taxa de prevalência de $31 \%$. A anemia, muitas vezes associada à malária, é um grave problema e em $68 \%$ das mulheres grávidas o Hematócrito situa-se abaixo de 33\%. (MISAU, 2009).

A busca ativa dos casos da malária clínica sugere que o risco da malária clínica é maior no grupo etário de 1 a 3 anos, quando as crianças podem experimentar uma média de mais de dois episódios por ano.

A resistência do $\mathrm{P}$. falciparum aos medicamentos antimaláricos, em especial ao tratamento de primeira linha da malária não complicada, com a cloroquina, varia entre 15 e 40\%, dependendo do local. A resistência aos medicamentos apresenta-se como um grande obstáculo para um manejo de casos eficaz, particularmente na periferia, onde a capacidade para o diagnóstico clínico e laboratorial é fraco. (MISAU, 2009).

\section{DISCUSSÃO}

Nota-se que no bairro 7 de setembro, na cidade de Chimoio, há um desinteresse na aderência da pulverização intra-domiciliária e este comportamento insatisfatório, tem contribuído bastante para a proliferação da malária.

Para o sucesso da implementação dos projetos de controlo da malária é imprescindível um conhecimento aprofundado dos fatores sociais e comportamentais que influenciam o risco da malária e que podem inibir ou facilitar modalidades particulares de intervenção, assim como posicionar o problema nos seus contextos sociais, económicos, políticos e culturais.

Embora as condições ambientais, a proximidade e exposição das pessoas aos parasitas ou vetores e os fatores microbiológicos e parasitológicos sejam extremamente importantes, e constituam fatores de risco imediatos, o comportamento das pessoas e a 
forma como os fatores socioculturais condicionam a sua vida são igualmente cruciais e constituem riscos fundamentais para a proliferação da doença.

A forma como a população se relaciona com os agentes infeciosos e com a gestão da doença, tem a ver com os padrões de conduta culturalmente codificados, com o sistema de normas e de valores que os orientam, assim como com o conhecimento construído e acumulado através dos vários recursos a que têm acesso, em conjunto com as experiências individuais e socialmente partilhadas. Neste sentido, é necessário perceber o contexto em que esta população está inserida, assim como as suas percepções, comportamentos e conhecimentos face à malária.

A maioria dos programas de controlo da malária implementados antes de 1990, não tinha em consideração o comportamento nem o sistema de valores das populações infetadas. Equipas formadas pelo governo entravam nos domicílios privados e desinfetavam-nos com inseticidas residuais (indoor residual insecticides), embora muitos dos residentes recusassem ou retirassem os sprays inseticidas das paredes tratadas após as intervenções (Vivek et al., 2003).

É já bastante reconhecido que a falta de atenção aos fatores socioculturais constituiu a maior razão para o insucesso dos esforços anteriores no controlo da Malária. Hoje, os programas são organizados horizontalmente e certas tendências etnocêntricas foram sendo postas de lado. O reconhecimento formal das diferenças, assim como a negociação de soluções mutuamente acordadas (pelas "adaptações" culturais), ajuda a formar a base de sucesso dos esforços pelo controlo da Malária.

A palavra percepção deriva do termo latino Perceptio, que se significa "compreensão", faculdade de perceber (Houaiss e Vilar, 2009). Este termo é definido, em linhas gerais, como o ato ou efeito de perceber, combinação dos sentidos no reconhecimento de um objeto, recepção de um estímulo, faculdade de conhecer independentemente dos sentidos, sensação e compreensão intelectual (Macete, 2016).

Ainda no mesmo desenvolvimento o autor refere que as pessoas diferem em sua percepção, pois a compreensão da experiência preceptiva é diferente de indivíduo no tempo e no espaço. Acrescenta ainda que a motivação pessoal, as emoções, os valores, os objetivos, os interesses as expectativas e outros estados mentais influenciam no que as pessoas percebem, a percepção pode ser entendida como um processo muito mais 
subjetivo do que se crê usualmente, é a representação a tomada de consciência das problemáticas ligadas ao ambiente, ou seja o ato de perceber o ambiente que está inserido, aprendendo a proteger e cuidar do mesmo (Oliveira e Corona, 2003).

Santos e Souza (2015), advogam que com a percepção ambiental se pode compreender melhor a inter-relação entre o homem e o meio ambiente, suas expectativas, anseios, satisfações, julgamentos e condutas perante o meio ambiente. Investiga a forma como o homem olha e interpreta, convive e se adapta à realidade do meio em que vive, principalmente quando se trata de ambientes instáveis e vulneráveis social e naturalmente (Silva e Bay, 2011).

Os autores ressaltam ainda que com a percepção ambiental aliada à educação ambiental, dotada de conhecimentos, valores e ações que os sujeitos possuem frente ao meio ambiente, facilita na elaboração de propostas, e estas podem atingir grande parte da sociedade, visando provocar mudança mais efetiva que contribua para a sustentabilidade sócio- ambiental.

O sector da saúde não pode, sozinho, melhorar a qualidade de vida das pessoas. Problemas ligados ao saneamento, escolarização, saúde condições trabalho entre outros constituem grandes dificuldades e só poderão encontrar soluções efetivas a partir de esforços de outros sectores da sociedade.

Libinga (2011) refere que a malária é uma doença parasitária cujo controlo não depende somente dos conhecimentos médicos, mas também das concepções populares sobre as causas e prevenção. O mesmo autor acrescenta que a educação passa a ser repensada como um processo capaz de desenvolver nas pessoas a consciência crítica das causas dos seus problemas e, ao mesmo tempo, criar prontidão para atuar na mudança. Ela tem a capacidade de gerar transformação de comportamentos das populações face a doenças como a malária.

De igual modo informações passadas através dos programas de saúde não chegam devidamente às populações. Essa informação não gera automaticamente uma rápida mudança de comportamento, talvez porque em alguns casos, a estratégia não se enquadre na realidade local.

Este tipo de atitude há vezes que promove informações reducionistas por alguns trabalhadores da saúde, considerando as populações como ignorantes (Libinga, 2011). 
Evidencia-se desta o papel da Educação Ambiental (EA). Esta que busca desenvolver uma população consciente e preocupada com o meio ambiente, com problemas que lhe são associados, visa transmitir conhecimentos, habilidades, atitudes, motivações e compromissos para trabalhar, individual e coletivamente na busca de soluções para os problemas existentes (Marcato, 2002).

\section{CONCLUSÃO}

Em Moçambique, os principais vetores da malária são endofágicos (alimentam-se no interior das casas) e, endofílicos (repousam dentro das casas), facto que torna estes vetores vulneráveis à pulverização intra-domiciliária com o uso de inseticidas (MISAU, 2006).

A disponibilização das intervenções-chave de controlo vetorial (redes com inseticida e/ou pulverização) e manejo de casos poderá não ser suficiente para a redução do peso da malária em Moçambique. A comunicação para a mudança social e de comportamento poderá desempenhar papel com importância similar e/ou maior que o controlo vetorial. Atuação sobre as redes sociais e comunitárias poderá ser uma importante porta de entradas das diversas metodologias e abordagens de comunicação para a mudança social e de comportamento.

Uma implementação com sucesso da estratégia dependerá da disponibilidade de recursos adicionais, mobilizados localmente e internacionalmente. O PNCM irá empreender-se para atrair fundos usando a estratégia como um instrumento para a mobilização de recursos. O Programa irá aproximar-se aos vários parceiros tradicionais e outros para angariar apoios para a implementação da estratégia e garantir a sustentabilidade das atividades de comunicação e advocacia.

Continua a ser importante e necessário apostar na formação dos indivíduos e continuar a realizar campanhas de esclarecimento e informação que capacitem as populações para o combate à doença que mais mata em todo o país. Tendo em conta a existência de grupos carenciados na população do Bairro 7 de Setembro-Chimoio, é possível que as pessoas estejam capacitadas ao nível de prevenção da malária, mas não o façam por não terem capacidade económica para adquirir os equipamentos necessários tais como mosquiteiros, redes para janelas e portas, repelentes, etc.

As formas mais eficazes de proteção contra as doenças transmissíveis continuam, porém, a ser as coletivas, e aqui cabe às autoridades de saúde pública o papel de maior protagonismo. Torna-se importante continuar a apostar na inclusão de conhecimentos de proteção da malária nos currículos escolares, desde o início da escolaridade e também 
oferecer formação adequada aos profissionais de saúde sobre o modo como fazer chegar essa informação às populações que se dirigem aos Hospitais e outros centros médicos.

As doenças de transmissão vetorial são consideradas hoje um problema crescente de saúde pública mundial, sobretudo nos países de clima tropical, onde as condições do meio ambiente favorecem o desenvolvimento e a proliferação do Aedes Aegypti, principal transmissor da doença.

\section{REFERÊNCIAS}

Bauren M, Zonatto VCS. Perfil dos artigos sobre controle interno no setor público em periódicos nacionais e internacionais. Rev. Adm. Pública, 2014; v.48, n.5. http://dx.doi.org/10.1590/0034-76121527

Cervo AL, Bervian PA. Metodologia Científica. 5ª Ed. São Paulo: Prentice Hall, 2002.

Fundamentação Teórica em Metodologia da Pesquisa, Unidade II, Capítulo I, Unyleya

Gil AC. Como Elaborar Projetos de Pesquisa. 4ª Ed. São Paulo: ATLAS, 78p. 2002

Libinga, L, J. (2011). Malária: Das representações sociais as lógicas terapêuticas nas zonas urbanas.

Marczwski M, Schwarzbold A, Machado NAF. Avaliação da percepção ambiental em uma população de estudantes do ensino fundamental de uma escola municipal rural: um estudo de caso [dissertação]. Instituto de Biociências. Porto Alegre (RS); 2006. http://hdl.handle.net/10183/8617

Ministry of Health National Malaria Strategic Plan 2012-2016, Ministry of Health National Malaria Control Programme, Maputo, Mozambique;

Ministry of Health, Malaria Indicator Survey (2007), Ministry of Health HQ, Maputo, Mozambique;

MISAU. Ministério da Saúde - Departamento de Epidemiologia e Endemias. Programa Nacional de Controlo da Malária - Documento Estratégico Para o Controlo Da Malária em Moçambique. Julho 2006-2009

Modelo do Projeto de Pesquisa. Unyleya;

Ribeiro JW, Rooke JMS. Saneamento Básico e sua relação com o meio ambiente e saúde pública. Revista Académica. Juiz de Fora.2010

Vivek, Pramila; Hackethal, Verónica; Heggenhougen, H. Kristian (2003), "The behavioural and social aspects of malária and its control", UNDP/ World Bank/ WHO Special Programme for Research and Training in Tropical Diseases (TDR). 